\title{
Self Evolving Neural Network Based Algorithm for Fault Prognosis in Wind Turbines : A Case Study
}

\author{
Pramod Bangalore \\ Chalmers University of Technology \\ Gothenburg, Sweden \\ pramod.bangalore@chalmers.se
}

\author{
Lina Bertling Tjernberg \\ KTH Royal Institute of Technology \\ Stockholm, Sweden \\ linab@kth.se
}

\begin{abstract}
Asset management of wind turbines has gained increased importance in recent years. High maintenance cost and longer downtimes of wind turbines have led to research in methods to optimize maintenance activities. Condition monitoring systems have proven to be a useful tool towards aiding maintenance management of wind turbines. Methods using Supervisory Control and Data Acquisition (SCADA) system along with artificial intelligence (AI) methods have been developed to monitor the condition of wind turbine components. Various researchers have presented different artificial neural network (ANN) based models for condition monitoring of components in a wind turbine. This paper presents an application of the approach to decide and update the training data set needed to create an accurate ANN model. A case study with SCADA data from a real wind turbine has been presented. The results show that due to a major maintenance activity, like replacement of component, the ANN model has to be re-trained. The results show that application of the proposed approach makes it possible to update and re-train the ANN model.
\end{abstract}

Index Terms-Artificial neural networks (ANN), condition monitoring (CMS), SCADA system, electricity generation.

\section{INTRODUCTION}

$\mathrm{W}$ IND industry has seen rapid growth in recent years with countries striving to have more sustainable energy sources in the electric power system. One of the obstacles for the growth of wind industry is high maintenance cost and long downtimes for wind turbines, especially for offshore wind farms. Wind turbine application to multi-megawatt electricity production is a developing technology. Developing technology poses challenges with respect to reliability of the wind turbines. Numerous efforts have been made towards efficient maintenance management of wind turbines in order to reduce maintenance cost and improve value of the asset [14].

Focus on early detection of failure of critical components in the wind turbine and condition based maintenance has increased in recent times. Traditional condition monitoring using vibration signals has proven to be a useful tool for monitoring the health of components. Furthermore, use of information rich SCADA data has received increased attention in recent years; see for example [5-8].

Artificial intelligence methods based on ANN have proven to be effective in modeling normal behavior characteristic of wind turbine components using SCADA data. An ANN model can mimic a non-linear relationship between input and output with good accuracy [9]. ANN needs to be trained before it can be used to model an input-output relationship. The training of ANN model is an important step, as the accuracy of the model will depend on the training data used. Traditionally training data set is chosen manually to cover all the operating points for the component; see for example [8].

This paper presents a case study for the approach to select the data set for training ANN model, presented in [10]. Data from a real wind turbine, rated $2 \mathrm{MW}$ and located in south of Sweden is used to demonstrate the application of the proposed approach. The approach will make the training of ANN model autonomous thereby avoiding any inadvertent error that might occur during manual selection of the training data set. Also, the automatic update will keep the ANN model up-to-date with respect to changing operating conditions of the wind turbine.

The paper is organized in following manner: Section II presents an introduction to neural networks and describes the neural network architecture used in this paper, Section III presents the proposed approach for using ANN for anomaly detection and the approach to update the training data set. Section IV presents a case study and results and Section V consists of discussion and conclusion.

\section{ARTIFICIAL NEURAL NETWORK}

Artificial neural network is based on the biological structure of the neural network in living organisms. Connection of simple processing units together enables delivery of a complex process. Fig. 1 shows a sample feed forward neural network. The dark circles are the inputs and output and the white circles are the neurons, each solid line represents a weight factor. Each input is multiplied by a weight factor and summed at each neuron, which is then passed through the transfer function, which decides output of each neuron. In this paper, a feed-forward neural network is

This project is financed through the Swedish Wind Power Technology Centre (SWPTC). SWPTC is a research centre for design of wind turbines. The purpose of the centre is to support Swedish industry with knowledge of design techniques as well as maintenance in the field of wind power. The Centre is funded by the Swedish Energy Agency, Chalmers University of Technology as well as academic and industrial partners. 
used with a sigmoid function for the first layer and a threshold function is for the second layer.

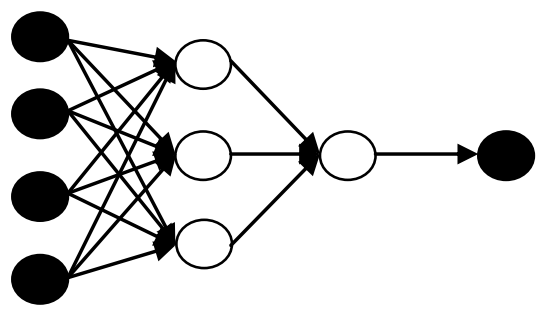

Fig. 1 Structure of feed-forward neural network

Fig. 2 shows a block diagram for supervised learning methodology. Learning is achieved by providing the information in the form of training data set to the neural network. The weights are decided based on the adopted training algorithm. In this paper Levenberg-Marquardt back propogation training algorithm is used, which updates the weights according to Levenberg-Marquardt optimization algorithm (LMA). LMA has the combined advantage of Newton's method, which converges rapidly and Gradient descent method, which is assured to converge [9].

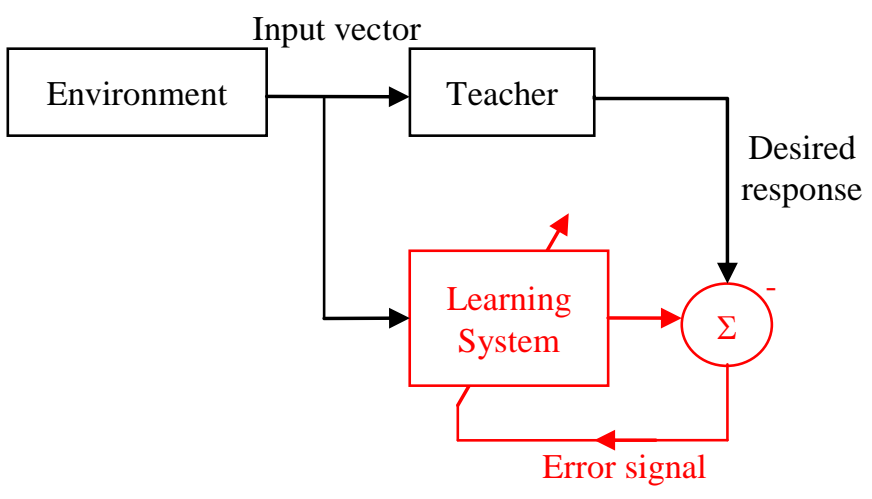

Fig. 2 Block diagram for supervised learning of the ANN [9]

A simplistic representative ANN structure is used in this paper for the purpose of demonstration. The ANN model has four layers; an input layer, two hidden layers and an output layer of neurons. The first hidden layer has 13 neurons and the second hidden layer has one neuron. This structure was decided after a few trial runs and it was found to give good performance.

The input/output relationship in the feed forward neural network can be described by the following set of equations:

$$
\begin{aligned}
& v_{\mathrm{k}}=\sum_{\mathrm{j}=1}^{\mathrm{n}} w_{k j} x_{j} \\
& \mathrm{y}^{\prime}(\mathrm{k})=\mathrm{S}\left(v_{\mathrm{k}}\right) \\
& \mathrm{y}=\theta\left(\sum_{\mathrm{k}=1}^{\mathrm{m}} \mathrm{y}^{\prime}(\mathrm{k})\right)
\end{aligned}
$$

$x_{1}, x_{2}, \ldots, x_{n}$ are input signals and $w_{k 1}, w_{k 2}, \ldots, w_{k n}$ are weight factors for neuron $\mathrm{k} \cdot \mathrm{y}^{\prime}(\mathrm{k})$ is the sigmoid combiner output due to the input signals. S (.) is the sigmoid activation function. $y$ is the output of the neural network obtained from a combiner due to the input to the second hidden layer and ' $m$ ' is the total number of neurons in second layer. $\theta($.$) is the$ threshold activation function.

\section{PROPOSED APPROACH}

\section{A. Approach for use of ANN for early fault detection}

The neural network can be used for early fault detection of critical wind turbine components. Fig. 3 shows the schematic of the approach using ANN model.

The trained ANN model is used to estimate the ten-minute average value of the gearbox bearing temperature based on the inputs. The estimated value is compared with the ten-minute average temperature stored in the SCADA system. The measured and the estimated gearbox bearing temperatures are compared. A deviation between the measured and the estimated values greater than a pre-set threshold will raise a flag, indicating deterioration in the bearing.

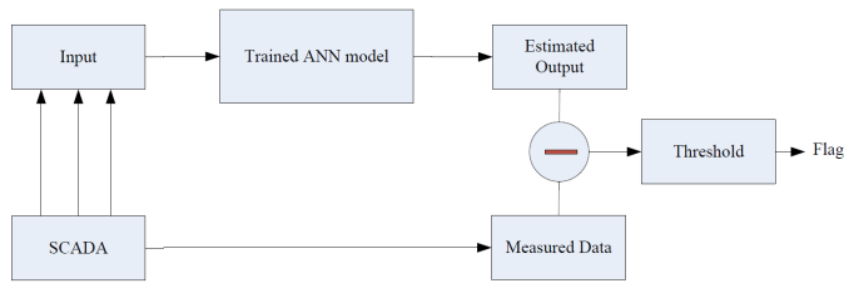

Fig. 3 Proposed structure to use ANN for anomaly detection for wind turbine gearbox bearing

The inputs and output used to model temperature behavior of the gearbox bearing are shown in Table I. The inputs selected have a direct effect on the behavior of the bearing temperature. Lubrication oil plays the role of taking the heat away from the bearings, whereas nacelle temperature will have a direct influence on bearing temperatures. Similarly turbine power and rpm, which decides the gearbox loading, have an effect on the temperature of the bearings.

TABLE I. INPUTS AND OUTPUT USED For ANN MODEL

\begin{tabular}{c|l}
\hline \multicolumn{1}{c|}{ Inputs } & \multicolumn{1}{|c}{ Output } \\
\hline Oil temperature & HSS (High \\
Nacelle temperature & $\begin{array}{l}\text { Speed Shaft) } \\
\text { Gear Bearing } \\
\text { Temperature }\end{array}$ \\
\hline Turbine Power & \\
\hline RPM &
\end{tabular}

Hence, the selected ANN model uses four inputs to give one output. Here the assumption is that at any given normal operating condition, there exists equilibrium between inputs and output. The aim is to model this equilibrium accurately. Under abnormal operating conditions the equilibrium is disturbed, which will lead to deviation between the model output and the actual measurement. This deviation will indicate deterioration in the monitored component, in this case the gearbox bearing.

\section{B. Selection of Threshold}

For detection of an anomaly it is important to set a correct threshold. In this paper, the method described in [7] is used to determine the threshold. The method is based on setting the threshold value based on the probability distribution of errors obtained during the training and testing stage of modeling. An error is defined as the difference between the estimated 
temperature from the ANN model and the actual temperature recorded in SCADA.

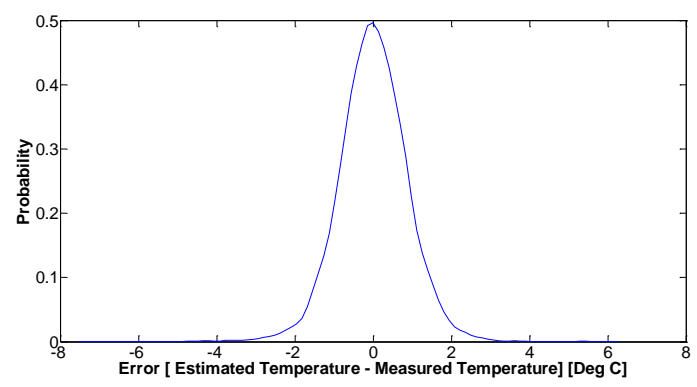

Fig. 4 Probability distribution of errors from training stage

Fig. 4 shows the probability distribution of the errors obtained during the training stage of modeling. The threshold value for detection of anomaly is decided based on the probability of occurrence of an error. In this paper, the threshold is set at $\mathrm{P}(\mathrm{e}) \leq 0.05$. The ANN model is used to estimate the temperature and the error is calculated as a root mean squared (RMS) value over a week. If the RMS error value has a probability of occurrence of less than 0.05 , then it is considered to be an anomaly in the component being monitored. However, with this threshold value there is a possibility of $5 \%$ false alarms. The rate of false alarms can be lowered by selecting a lower threshold, for example $1 \%$ probability.

\section{Approach for selection of training data set}

An approach for selection of training dataset is presented in [10]. The approach is briefly presented in this section.

Fig. 5 shows the gearbox bearing 10-min SCADA recorded temperature measurement averaged over a period of three months for five different wind turbines. It can be inferred from Fig. 5 that each wind turbine has its own range of operating conditions. This individuality is referred to as the 'behavior profile' of the wind turbine, in this paper. Due to this individuality, separate models need to be created for each wind turbine. The automated process of data selection aids in selecting data based on the behavior profile of the wind turbine.

A diversity measure ' $D M$ ', which shows the difference in data from two seasons is calculated using Eq.4.

$\forall i, j \leq n \& i \neq j ; D M=\arg _{\max }|| \tau_{\mathrm{av}_{\mathrm{i}}}-\tau_{\mathrm{av}_{\mathrm{j}}} \|$

$\tau_{\mathrm{av}}$ is the average gearbox bearing temperature calculated over a period of three months of the same season and ' $n$ ' is the number of seasons.

The final objective is to pick data sets from two months, which show maximum diversity, so that highest number of operating points is covered. The initial training data set is selected when ' $D M$ ' has maximum value.

Considering Turbine A1 from Fig. 5 and Eq.4, it was found that the data sets from winter and summer months present the most diverse data. Hence, the initial training data set consists of one month data from winter and one month data from summer months. Data from any month can be selected once the season of interest is determined. Here data from the first winter month and the first summer month are selected to create the initial training data set.

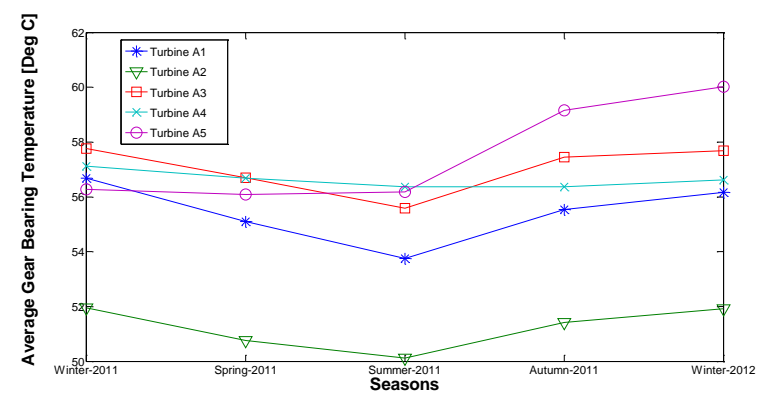

Fig. 5 Behavior profile for wind turbines A1 to A5

To finalize the training data set more data points are added to the initial training data set and this addition is controlled as per Eq. 5.

$\left[\left(\mathrm{A}_{\max }-\mathrm{B}_{\max }\right)+\left(\mathrm{B}_{\min }-\mathrm{A}_{\min }\right)\right]+N \leq \varepsilon$

$A_{\text {max }}$ is a vector of maximum values of input parameters in actual data set which includes data from one year. Referring to Table I, vector $A_{\max }$ can be decided as follows:

$A_{\max }=\left[\right.$ Oiltemp $_{\text {max }}^{\text {Act }}$, Nactemp $_{\text {max }}^{\text {Act }}$, Power $\left._{\text {max }}^{\text {Act }}, R P M_{\text {max }}^{\text {Act }}\right]$

The 'max' values in Eq.6 denote the maximum value of the parameter in data set containing data from one year.

Similarly, $B_{\max }$ is the vector of maximum values of input parameters in training data set. $A_{\min }$ and $B_{\min }$ are vectors of minimum values of input parameters in actual and training data sets respectively. The value $N$ is the number of sample points in the training data set and $\varepsilon$ is called a control parameter. The control parameter limits the number of sample points to a maximum value. In the case study described in this paper, the value of control parameter ' $\varepsilon$ ' is chosen to be 8500 . This value is selected so that the training data set includes at least two month data.

\section{Approach for updating ANN Model}

Wind turbine operation and maintenance providers, generally, follow a six month preventive maintenance regime. These timely maintenance activities keep the operating condition of the wind turbine within the normal operating range.

The gearbox temperature displays a cyclic characteristic, which is season dependant. With no degradation or fault in the gearbox the variation is within some limits as seen in Fig. 6.

However, if there is a replacement of a major component like the gearbox, the operating range for the wind turbines can alter considerably. Hence, it becomes necessary to update the ANN model with new data, in order to avoid false alarms. An approach is presented, which considers major maintenance actions, like replacement of a critical component, and updates 
the ANN model. The aim is to keep the ANN model up-todate by re-training after certain maintenance actions. To make this updating procedure automated the approach presented in Fig. 7 is proposed.

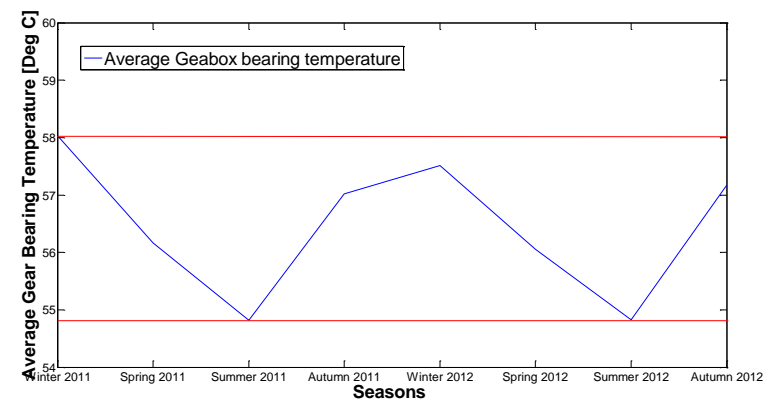

Fig. 6 Average gearbox bearing temperature for a turbine shown over a period of two years

In line with the proposed approach, after a major replacement, there exists a waiting period of 90 days, during which the ANN based condition monitoring is not available. The data from this period is used to set the baseline of the operating characterisitcs of the new component. During this time it is considered that other types of condition monitoring like vibration based condition monitoring are available and will aid in continued monitoring of the component.

\section{CASE STUdy}

In this section results from a case study are presented. The ANN model is used for anomaly detection. The case study is applied to data from a real wind turbine SCADA system. The wind turbine is located onshore and has a rating of $2 \mathrm{MW}$. A gearbox failure was recorded in the wind turbine under consideration. The updating approach is demonstrated with data before and after the replacement of the gearbox.

\section{A. Selection of training data set}

The proposed approach for automated selection of training data set was applied to SCADA data from one year operation of a wind turbine. The data was extracted from the year 2010, as there was no recorded failure in the gearbox. In order to ensure the entire range of data has been covered in the training data set, the probability distributions of the data from entire year are compared to the data from selected training data set.

From Fig. 8 to 12 , it can be seen that in all the cases the entire range of data is covered.

In this paper, batch training algorithm is used for training the neural networks. Batch training has the advantage of parallelization of learning process, which makes the training faster [9]. However, if the batch training is applied, repetitive training samples do not contribute towards improving the performance of the ANN. Hence, with regards to accuracy of the ANN model, the data from two months, which covers the operating range for wind turbines is considered sufficient instead of using the data from entire year. This will improve the training speed in the ANN modeling process.

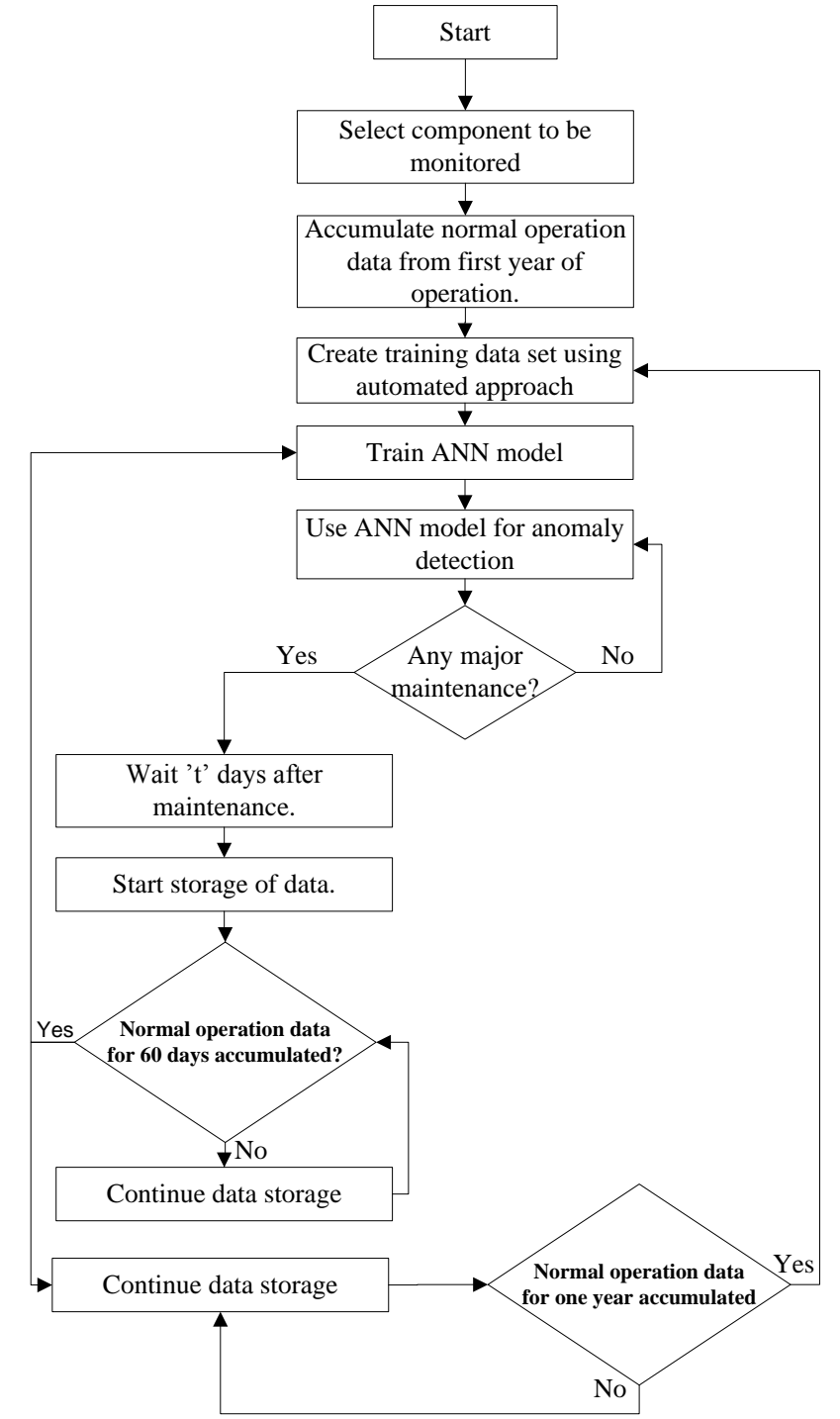

Fig. 7 Proposed approach for automatic update of the ANN model

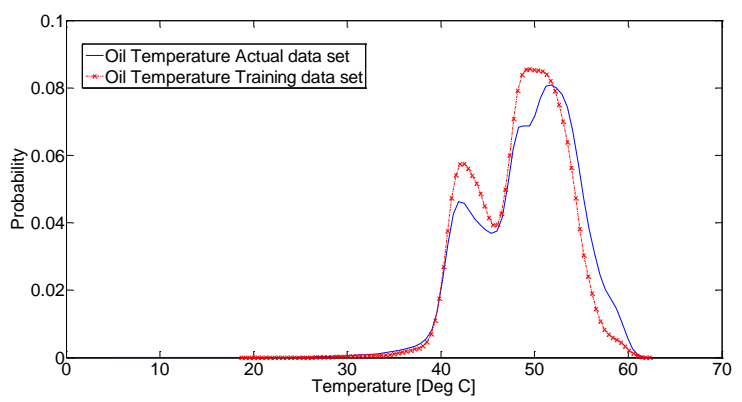

Fig. 8 Probability distributions for Oil Temperature from actual data set and training data set 


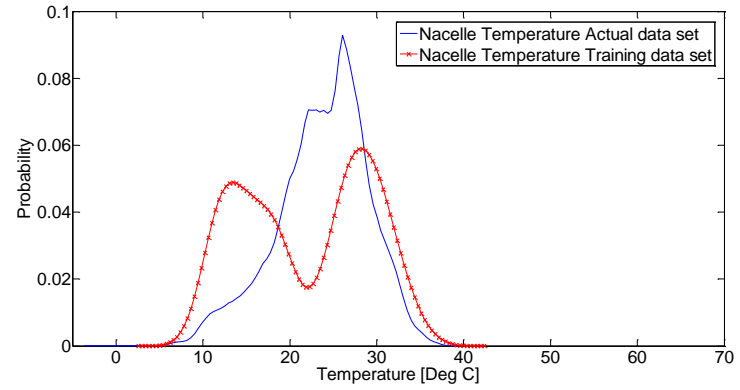

Fig. 9 Probability distributions for Nacelle Temperature from actual data set and training data set

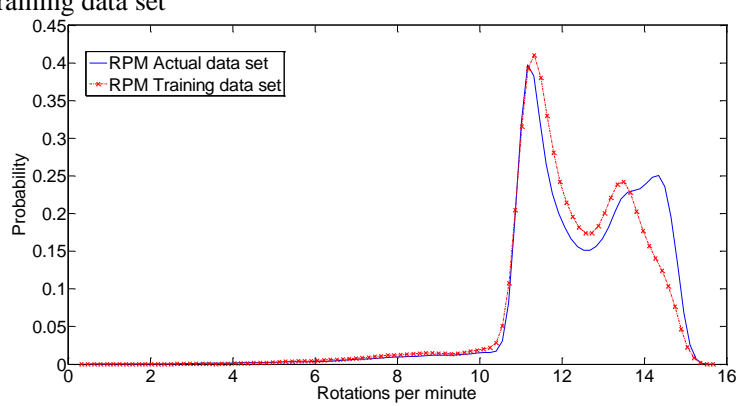

Fig. 10 Probability distribtuions for RPM from actual data set and training data set

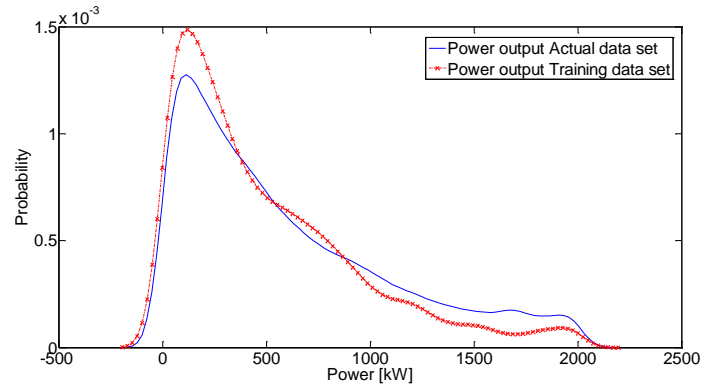

Fig. 11 Probability distributions for Power produced from actual data set and training data set

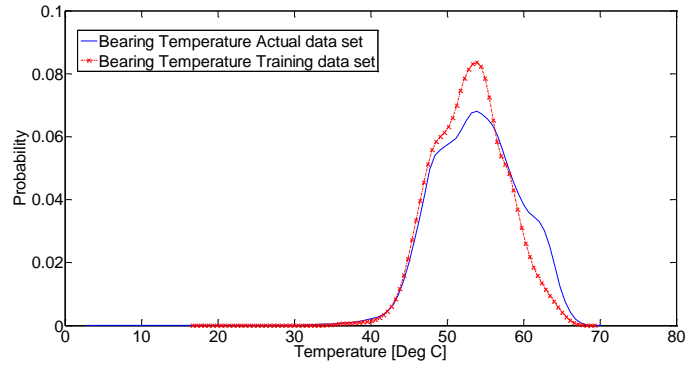

Fig. 12 Probability distributions for Bearing Temperature from actual data set and training data set

\section{B. ANN model for anomaly detection}

In [10], it was shown that the accuracy of the ANN model obtained by using the proposed automated data selection approach is approximately the same compared to the conventional approach of manual selection of training data set. The ANN model trained with the selected training data set is used for anomaly detection, as discussed in previous sections.
Fig. 13 shows the weekly RMS value of error between the estimated bearing temperature and the actual measured bearing temperature.

As seen in Fig. 13, the error between the estimated and the actual temperature has crossed the threshold value at the end of Jan 2012. At this time, a gear in the gearbox was broken and the gearbox was replaced. The ANN model is used to monitor the gearbox bearing. However, due to severe abnormal conditions due to broken gear, the effect had propagated to the bearings and the model could detect the error. It can also be observed that during week 42 the error has crossed the threshold. An inspection at this stage could have lead to detection of the degradation in the gears.

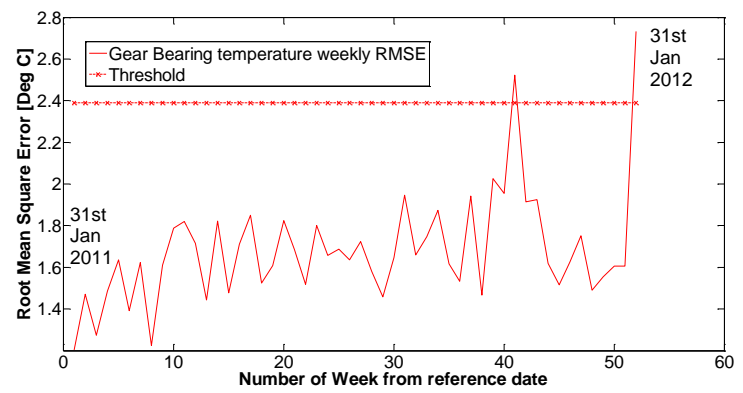

Fig. 13 Weekly RMS error between estimated and actual bearing temperatures

\section{Updating procedure: Case study}

After replacement of the gearbox in the wind turbine, it was noticed that the operating behavior of the gearbox bearings had changed.

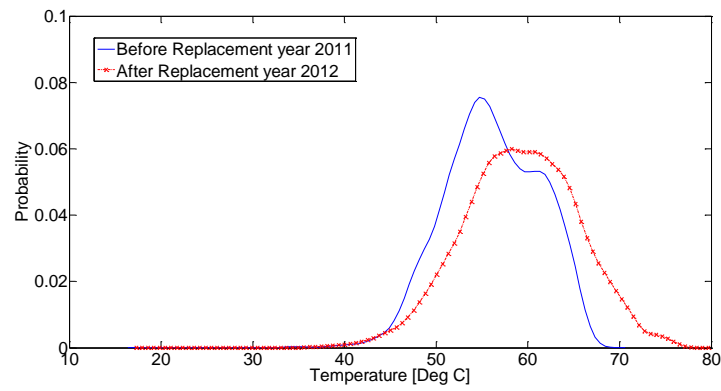

Fig. 14 Probability distribution of gearbox bearing temperature before and after gearbox replacement

Fig. 14 shows the comparison of probability distribution of gearbox bearing temperature for the time frames January to May for years 2011 and 2012. In Feb 2012 the gearbox was replaced after a failure and the behavior profile of the gearbox bearing changed considerably. It can be seen that the normal operating temperature for the gearbox bearing is much higher after replacement. Fig. 15, shows the error between the estimated and actual measured bearing temperatures when the old ANN model is used.

Fig. 15, shows the ANN model output for six months starting from $1^{\text {st }}$ May 2012 to $31^{\text {st }}$ October 2012. It can be seen that the operation of the gearbox bearing is out of normal 
during this time. However, this out of normal operation should be considered as a false alarm as there was no recorded fault during this period. This means that the ANN model trained on data from the year 2010 is not suitable anymore to detect anomalous operation after the gearbox has been replaced. In this situation, the approach proposed in Fig. 7 is applied to update the ANN model. Hence, in line with the proposed approach there was a waiting time of 4 weeks. The data from month of February 2012 was not considered for the training. The data for next 60 days, that is, data from March and April 2012 has been considered to train the new ANN model. This is used to train a temporary ANN model, which can be used for anomaly detection.

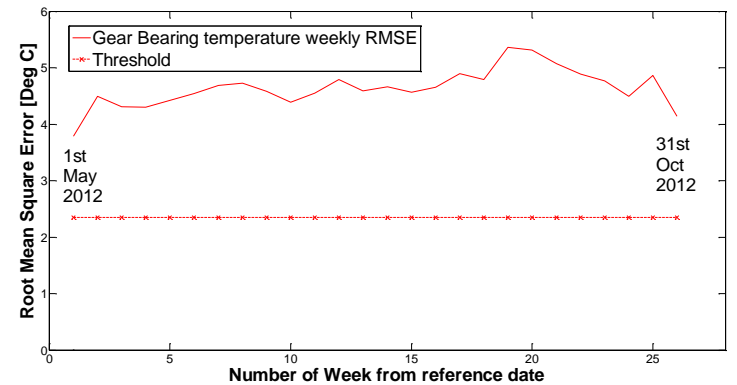

Fig. 15 Weekly RMS error between estimated and actual bearing temperatures

Fig. 16 shows the updated ANN model output compared to the actual measured temperature for the same period, $1^{\text {st }}$ May 2012 to $31^{\text {st }}$ October 2012. The operation of the gearbox bearing is within the normal operating range. Hence, the updated model is able to model the behavior of the gearbox bearing after replacement of the gearbox. Furthermore, as presented in the approach, after un-faulted data from one year operation has been gathered, re-training is initiated to update the ANN model.

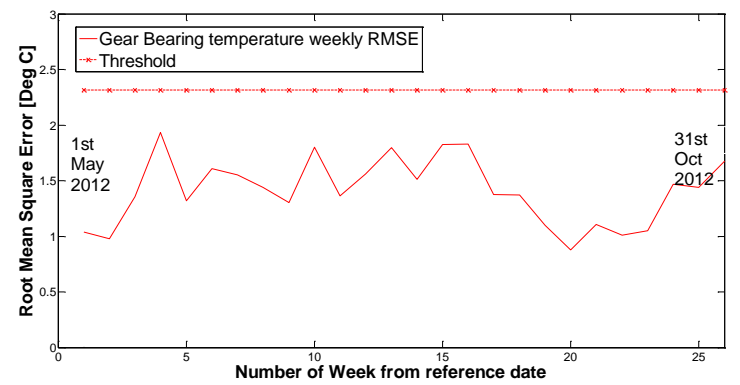

Fig. 16 Weekly RMS error between estimated and actual bearing temperatures

\section{DisCUSSION AND CONCLUSION}

Artificial intelligence (AI) methods like ANN give an opportunity to utilize the data stored in SCADA to monitor behavioral changes in some critical components in the wind turbine. Researchers have developed various ANN based models for condition monitoring of components. Training is a crucial stage in ANN modeling. However, this topic is not addressed frequently. This paper has presented a generalized approach to select training data set for ANN modeling for wind turbine fault detection, which can be used irrespective of the structure of ANN.

Major maintenance activities, like replacement of a gearbox, can change the behavior significantly. There is a need to review these changes and up-date the ANN model in order to continue use of AI method to monitor the component. The proposed updating procedure for the ANN model can take this information in to account once feedback from the operator about maintenance is received.

From the presented case study results it can be concluded that there is a need to review and make changes in the ANN model after a major maintenance activity and the proposed approach is capable to update the ANN model as required.

\section{ACKNOWLEDGMENT}

The authors would like to kindly acknowledge the partnering companies, Triventus AB, Göteborg Energi and SKF for their support and guidance during this work.

\section{REFERENCES}

[1] J. A. Andrawus, J. Watson, and M. Kishk, "Modelling system failures to optimise wind turbine maintenance," Wind Engineering, vol. 31, pp. 503-522, 2007.

[2] J. A. Andrawus, J. Watson, and M. Kishk, "Wind turbine maintenance optimisation: Principles of quantitative maintenance optimisation," Wind Engineering, vol. 31, pp. 101-110, 2007.

[3] K. Fischer, F. Besnard, and L. Bertling, "Reliability-Centered Maintenance for Wind Turbines Based on Statistical Analysis and Practical Experience," IEEE Transactions on Energy Conversion,, vol. 27, pp. 184-195, 2012.

[4] F. Besnard and L. Bertling, "An Approach for Condition-Based Maintenance Optimization Applied to Wind Turbine Blades," IEEE Transactions on Sustainable Energy, vol. 1, pp. 77-83, 2010.

[5] M. C. Garcia, M. A. Sanz-Bobi, and J. del Pico, "SIMAP: Intelligent System for Predictive Maintenance. Application to the health condition monitoring of a windturbine gearbox," Computers in Industry, vol. 57, pp. 552-568, 2006.

[6] K. Kim, G. Parthasarathy, O. Uluyol, W. Foslien, S. Sheng, and P. Fleming, "Use of SCADA Data for Failure Detection in Wind Turbines," ASME Conference Proceedings, vol. 2011, pp. 2071-2079, 2011.

[7] M. Schlechtingen, I. F. Santos, and S. Achiche, "Wind turbine condition monitoring based on SCADA data using normal behavior models. Part 1: System description," Applied Soft Computing Journal, vol. 13, pp. 259-270, 2013.

[8] A. Zaher, S. D. J. McArthur, D. G. Infield, and Y. Patel, "Online wind turbine fault detection through automated SCADA data analysis," Wind Energy, vol. 12, pp. 574-593, 2009.

[9] S. S. Haykin, Neural networks and learning machines, 3. ed. Upper Saddle River: Pearson Education, 2009.

[10] P. Bangalore and L. Bertling, "An Approach for Self Evolving Neural Network Based Algorithm for Fault Prognosis in Wind Turbine," In proceeding of IEEE PowerTech Conference, Grenoble, 2013. 